\title{
Aspectos da vegetação e estado de conservação de nascentes do rio Piauitinga, Sergipe-Brasil
}

\author{
Robério Anastácio Ferreira ${ }^{1 *}$ Marx Iuri Costa Nascimento ${ }^{2}$ Dráuzio Correia Gama ${ }^{3}$ Thadeu Ismerim \\ Silva Santos ${ }^{4}$ Diogo Gallo de Oliveira ${ }^{5}$ Airon José da Silval Anabel Aparecida de Mello ${ }^{1}$ \\ ${ }^{1}$ Universidade Federal de Sergipe, Av. Marechal Rondon S/N, São Cristóvão, SE, Brasil \\ ${ }^{2}$ Engenheiro Florestal, Brasil \\ ${ }^{3}$ Mestre em Ciências Florestais, Brasil \\ ${ }^{4}$ Projeto Azahar: Flor de Laranjeiras - Fundação de Apoio à Pesquisa e Extensão de Sergipe (Fapese), Brasil \\ ${ }^{5}$ Universidade Federal do Vale do São Francisco, Núcleo de Ecologia e Monitoramento Ambiental (Univasf/Nema), Petrolina, PE, Brasil.
}

\begin{abstract}
Artigo original
*Autor correspondente: roberioaf@yahoo.com.br

Palavras-chave:

Vegetação ripária

Restauração ambiental

Composição florística

Fitossociologia

Keywords:

Riparian vegetation

Environmental restoration

RESUMO: A Sub-Bacia Hidrográfica do rio Piauitinga é uma área que vem sofrendo diversas transformações ambientais ao longo do tempo em consequência de constantes desmatamentos. O presente trabalho foi realizado com a finalidade de conhecer a composição florística arbóreo-arbustiva, a estrutura fitossociológica e identificar usos e ocupação dos solos das áreas de nascentes do alto curso do rio Piauitinga, situadas no município de Lagarto - SE. As análises foram realizadas em um raio de $50 \mathrm{~m}$ no entorno das nascentes, por meio de censo, contabilizando todos os indivíduos arbustivo-arbóreos vivos com diâmetro à altura do peito (DAP a 1,30 $\mathrm{m}$ do solo) $\geq 5,0 \mathrm{~cm}$. Foram medidos 1.550 indivíduos, distribuídos em 76 espécies, 58 gêneros e 36 famílias botânicas, em 26 nascentes. Os índices de diversidade ( $H^{\prime}$ ) e equabilidade ( $\mathrm{J}$ ') encontrados foram de 3,04 nats.ind ${ }^{-1}$ e 0,71 , respectivamente. A similaridade florística (SJ) variou de $0,08 \%$ a $56,2 \%$. A densidade total foi de 98,73 ind.ha- ${ }^{-1} \mathrm{e}$ a área basal de $68,46 \mathrm{~m}^{2} \cdot \mathrm{ha}^{-1}$. Verificou-se apenas uma nascente em estado de conservação preservada, seis perturbadas e 19 degradadas. Com 22 nascentes pontuais e quatro difusas, ocupadas principalmente por áreas agrícolas e de pastagens.
\end{abstract}

Floristic composition

Phytosociology

Received in

$2020 / 01 / 18$

Accepted in

2021/12/06

Published in

2021/12/30

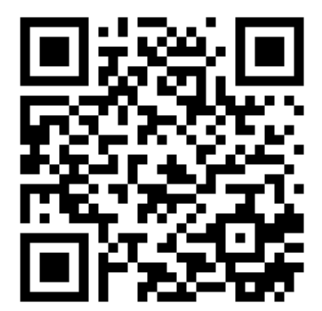

DOI:

https://doi.org/10.34062/afs. v8i4.9699

\section{(cc) BY}

\section{Vegetation aspects and conservation status of Piauitinga river springs, Sergipe-Brazil}

\begin{abstract}
The River Piauitinga Hydrographic Sub-Basin is an area that has undergone several environmental changes over time as a result of constant deforestation. The present work was performed with the purpose of knowing the tree-shrub floristic composition, the phytosociological structure and identifying uses and occupation of the soils in the headwaters areas of the upper Piauitinga River, located in the municipality of Lagarto - SE. The analyzes were carried out in a 50m radius around the springs, by means of a census, counting all live shrub-tree individuals with a diameter at breast height (DBH at $1.30 \mathrm{~m}$ from the ground) $\geq 5.0$ $\mathrm{cm}$. 1,550 individuals were measured, distributed in 76 species, 58 genera and 36 botanical families in 26 springs. The diversity $\left(\mathrm{H}^{\prime}\right)$ and equability $\left(\mathrm{J}^{\prime}\right)$ indexes found were 3.04 nats.ind $^{-1}$ and 0.71 , respectively. Floristic similarity (SJ) ranged from $0.08 \%$ to $56.2 \%$. The total density was 98.73 ind.ha $^{-1}$ and the basal area was 68.46 $\mathrm{m}^{2}$.ha ${ }^{-1}$. There was only one spring in a preserved state, six disturbed and 19 degraded. With 22 point springs and four diffuse springs, occupied mainly by agricultural and pasture areas.
\end{abstract}




\section{Introdução}

A cobertura vegetal de uma Bacia Hidrográfica tem grande valor por exercer importantes funções como a proteção de corpos d'água e do solo contra processos erosivos, bem como a formação de corredores ecológicos que visam manter um fluxo gênico tanto da flora quanto da fauna na região (Brasil 2012a, Tambosi et al. 2015, Leal et al. 2017).

Por outro lado, a proteção das matas ciliares de nascentes reflete diretamente na qualidade de suas águas produzidas, segundo autores como Marmontel e Rodrigues (2015), relacionada com a dependência dessa qualidade ao consumo humano e a dessedentação animal, por exemplo (Bomfim et al. 2015, Honda e Durigan 2017).

Quanto a degradação de nascentes, essa se deve diretamente as atividades agropecuárias (Silva et al. 2016), resultando numa substituição progressiva da vegetação nativa por uma paisagem cada vez mais heterogênea e fragmentada (Coelho et al. 2014), afetando a redução da disponibilidade hídrica, expressa em baixa vazão da nascente ou curso d'água (Vanzela et al. 2010, Pereira-Silva et al. 2011), causando ainda perda de biodiversidade e desequilíbrio dos processos ecológicos (Brasil 2012a, Siqueira et al. 2016).

$\mathrm{Na}$ Bacia Hidrográfica do rio Piauitinga, no estado de Sergipe, por exemplo, aproximadamente $70 \%$ das áreas de preservação permanente (APP), são ocupadas por outras formas de uso do solo, sobressaindo-se o domínio das pastagens em 50,39\% e com 24\%, aproximadamente, de área com vegetação nativa conservada (Santos et al. 2017). Desse modo, visando à recuperação de APP, em especial em áreas de matas ciliares, a realização de estudos sobre a composição florística e estado de conservação das nascentes, por exemplo, contribuem na construção de fundamentações teóricas à conservação dos recursos genéticos e áreas similares à recuperação (Sobrinho et al. 2009). De acordo com Paula et al. (2004) a estimativa de parâmetros fitossociológicos, somada a uma listagem florística e dos grupos ecológicos, tornam-se ferramentas indispensáveis para o auxílio de estudos da vegetação.

Estudos esses, que também auxiliam nas possibilidades de classificação dos estados de conservação (preservadas, perturbadas ou degradadas) das áreas de nascentes e seus afloramentos na superfície em tipos pontual ou difusa em vários pontos diferentes (Pinto et al. 2005a)

Diante do exposto, o presente trabalho tem o objetivo de conhecer e mensurar a composição florística arbóreo-arbustiva, a estrutura fitossociológica e identificar usos e ocupação dos solos das áreas de nascentes do alto curso rio Piauitinga, situadas no município de Lagarto-SE.

\section{Material e Métodos}

Caracterização da área de estudo

As nascentes estudadas localizam-se no município de Lagarto, situado no centro-sul do estado de Sergipe, inseridas na Sub-Bacia Hidrográfica do rio Piauitinga. Delimitadas pelas coordenadas geográficas: $10^{\circ} 34^{\prime} 10^{\prime \prime} ; 10^{\circ} 45^{\prime} 12^{\prime \prime} \mathrm{S}$ e $37^{\circ} 22^{\prime} 20^{\prime \prime} ; 37^{\circ} 34^{\prime} 22^{\prime \prime} \mathrm{W}$ (Figura 1).

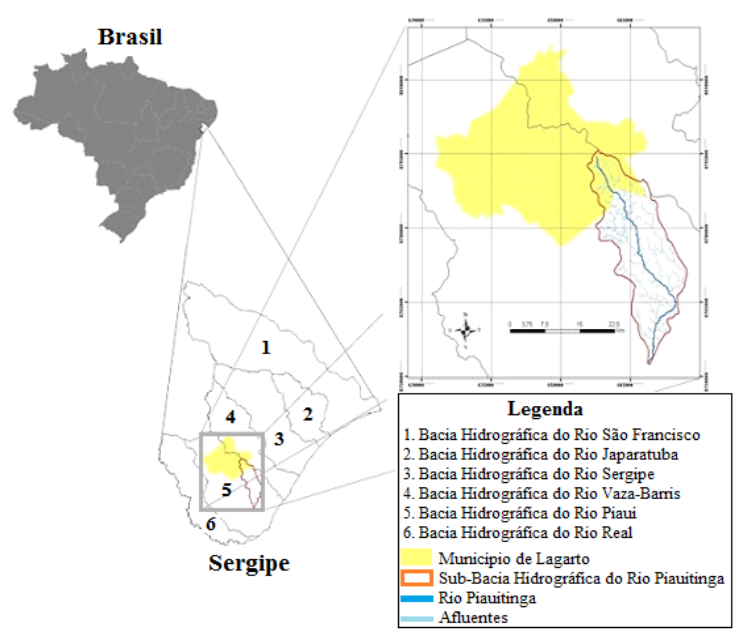

Figura 1. Localização da área de estudo no alto-curso do rio Piauitinga no município de Lagarto, Sergipe. Fonte: Adaptado de Sergipe (2004).

O clima do município é do tipo Aws, caracterizado como tropical savânico, com inverno e verão seco (Alvares et al. 2013). A precipitação média anual é de $1.067 \mathrm{~mm}$, com chuvas concentradas entre abril a julho e de outubro a janeiro. A temperatura média é de $24,0{ }^{\circ} \mathrm{C}$, sendo fevereiro o mês mais quente do ano com média de $25,6{ }^{\circ} \mathrm{C}$ e julho o mais frio com média de $21,7{ }^{\circ} \mathrm{C}$ (Climate-Data 2012).

A formação florestal da região de Lagarto caracteriza-se por áreas de tensão ecológica (ecótonos), localizada entre a Floresta Estacional Semidecidual do Domínio Fitogeográfico de Mata Atlântica e Savana (Brasil 2012b).

As áreas do entorno das nascentes distribuem-se em posições erosicionais (quebra-derelevo), baixadas com hidromorfismos e sopé de encosta suave de topo de tabuleiros costeiros. Predominam solos do tipo Gleissolo Háplico e Gleissolo Melânico em relevo plano a forte e suaveondulado; Cambissolo Háplico Gleissólico e Cambissolo Háplico Petroplíntico em relevo ondulado a forte-ondulado; Plintossolo Háplico em relevo plano a suave-ondulado e Argissolo Acinzentado em relevo plano sopé de encosta (Magalhães et al. 2012).

A Sub-Bacia Hidrográfica do rio Piauitinga compreende os municípios de Boquim, Estância, Lagarto, Salgado e Itaporanga d’Ájuda em uma área 
de 411,98 $\mathrm{km}^{2}$ e perímetro de 121,22 km (Aguiar Netto et al. 2015). Essa Sub-Bacia Hidrográfica é tributária da Bacia Hidrográfica do rio Piauí ao qual abrange uma área de 3.930,67 km² e população residente total de 335.317 habitantes, associada às atividades econômicas, ligada aos setores privado e público, possibilitando o desenvolvimento regional por disponibilizar água aos diversos usos (irrigação, mineração, industrial, consumo humano e animal, pesca, turismo e lazer) com demanda hídrica de 24,68 milhões de $\mathrm{m}^{3}$.ano ${ }^{-1}$, predominando o abastecimento humano (Sergipe 2010).

\section{Amostragem e classificação das nascentes}

Por método de caminhamento e com auxílio de moradores locais, foram localizadas 26 nascentes, classificadas e em seguida georreferenciadas com a utilização de aparelho GPS (Garmim MAP 76 CSx), no período de julho de 2006 a fevereiro de 2007.

Considerando-se a cobertura vegetal existente num raio de $50 \mathrm{~m}$ do entorno de uma nascente (Brasil 2012a), as nascentes foram classificadas em preservadas, quando a vegetação medida a partir do olho d'água foi igual ou superior ao raio de $50 \mathrm{~m}$ no seu entorno; em perturbadas, quando não possuíam vegetação no raio de $50 \mathrm{~m}$ no seu entorno e em degradadas, quando possuíam muito pouco de vegetação e encontrava-se com alto grau de degradação e com solo compactado, presença de gado, erosão, construção de pequenas barragens, despejo de esgotos e outros dejetos (Faria et al. 2012, Brasil 2013). Quanto ao fluxo de água, as nascentes foram classificadas em perenes (fluxo único e contínuo), inclusive na estação seca e intermitentes (fluxo durante a estação das chuvas), com base em Machado et al. (2018).

\section{Inventário florestal, levantamento florístico $e$ classificação sucessional}

Para as análises das espécies arbóreoarbustivas foi realizado um inventário florestal utilizando-se do censo no entorno das nascentes utilizando um raio de $50 \mathrm{~m}$ (0,785ha). Foram registrados e mensurados todos os indivíduos de espécies nativas e exóticas com DAP (diâmetro à altura do peito medido a $1,30 \mathrm{~m}$ do solo) $\geq 5 \mathrm{~cm}$. Os indivíduos bifurcados também foram considerados, desde que pelo menos uma das ramificações atendesse ao critério de inclusão.

O material botânico foi coletado durante a realização do levantamento da vegetação, dando-se preferência aos indivíduos que apresentavam estruturas reprodutivas (flor e fruto), sendo confeccionadas exsicatas para herborização e, posteriormente, identificação por comparação com exsicatas existentes no Herbário da Universidade Federal de Sergipe (ASE), no Instituto do Meio Ambiente-AL (MAC) e, também, por consulta em literatura especializada. Posteriormente, o material botânico foi incorporado ao Herbário da Universidade Federal de Sergipe (ASE).

A confirmação da nomenclatura taxonômica foi realizada a partir de consulta ao banco de dados da lista de espécies da Flora do Brasil 2020 (Brasil 2020). Quanto ao estádio sucessional, as espécies foram classificadas em grupos ecológicos, sendo eles: i) pioneiras (P); ii) clímax exigente em luz (CL); iii) clímax tolerante à sombra (CS), conforme Swaine e Whitmore (1988).

\section{Análise fitossociológica e índices estimativos}

$\mathrm{Na}$ análise fitossociológica foi utilizado os parâmetros de estrutura horizontal: densidade absoluta (DA), frequência absoluta (FA), dominância absoluta (DoA), densidade relativa (DR), frequência relativa (FR), dominância relativa (DoR) e o valor de importância (VI), conforme Chaves et al. (2013), com o auxílio do Software "Mata Nativa 2" licenciado para o Departamento de Ciências Florestais da Universidade Federal de Sergipe ${ }^{\circledR}$ (Cientec 2006).

$\mathrm{Na}$ estimativa da diversidade florística entre as nascentes, utilizou-se o índice de diversidade de Shannon-Weaver (H'). A uniformidade de distribuição dos indivíduos entre todas as espécies existentes foi determinada pelo índice de equabilidade de Pielou (J'), que leva em consideração o intervalo de 0 a 1 , onde 1 representa a máxima diversidade. E a similaridade florística foi determinada pelo coeficiente de Jaccard (SJ), conforme Brower e Zar (1984) e Magurran (1988).

\section{Uso e ocupação dos solos}

Nas áreas do entorno das nascentes foram também observados os principais usos e ocupação dos solos pelas comunidades circunvizinhas e proprietários rurais onde as nascentes estão situadas. Para esta finalidade, foram utilizadas as categorias de uso e ocupação de solos conforme Manual Técnico de Uso da Terra (Brasil 2013).

\section{Resultados e Discussão}

Das 26 nascentes encontradas na Sub-Bacia Hidrográfica do rio Piautinga, no município de Lagarto, apenas uma foi classificada como preservada $(3,84 \%)$, sendo $19 \quad(73,07 \%)$ como degradadas e seis $(23,07 \%)$ em estado de conservação perturbada (Tabela 1). 
Ferreira et al.

Tabela 1. Classificação das nascentes situadas no município de Lagarto-SE, na Sub-Bacia Hidrográfica do rio Piauitinga, quanto ao tipo de reservatório, estado de conservação e fluxo de água. Em que: Nasc. - Nascente; Lat. - Latitude; Long. - Longitude; Alt. (m) - Altitude em metros; Pt - pontual; Di - difusa; Pr - preservada; Pe perturbada; Dr - degradada; Pn - perene; In - intermitente.

\begin{tabular}{|c|c|c|c|c|c|c|c|c|c|c|}
\hline \multirow{2}{*}{ Nasc. } & \multirow{2}{*}{ Lat. } & \multirow{2}{*}{ Long. } & \multirow{2}{*}{$\begin{array}{l}\text { Alt. } \\
\text { (m) }\end{array}$} & \multicolumn{2}{|c|}{ Reservatório } & \multicolumn{3}{|c|}{ Conservação } & \multicolumn{2}{|c|}{ Fluxo } \\
\hline & & & & $\mathbf{P t}$ & Di & Pr & $\mathbf{P e}$ & Dr & Pn & In \\
\hline 1 & 656471 & 8792662 & 164,0 & $\mathrm{x}$ & - & - & - & $\mathrm{x}$ & $\mathrm{x}$ & - \\
\hline 2 & 656491 & 8792657 & 164,0 & $\mathrm{x}$ & - & - & - & $\mathrm{x}$ & $\mathrm{x}$ & - \\
\hline 3 & 657457 & 8792942 & 160,3 & $\mathrm{x}$ & - & - & - & $\mathrm{x}$ & $\mathrm{x}$ & - \\
\hline 4 & 657781 & 8792952 & 160,9 & $\mathrm{x}$ & - & - & - & - & $\mathrm{x}$ & - \\
\hline 5 & 658084 & 8790842 & 152,0 & - & $\mathrm{x}$ & - & $\mathrm{x}$ & - & $\mathrm{x}$ & - \\
\hline 6 & 658020 & 8790945 & 159,0 & $\mathrm{x}$ & - & - & $\mathrm{x}$ & - & $\mathrm{x}$ & - \\
\hline 7 & 659525 & 8791221 & 151,0 & $\mathrm{x}$ & - & - & - & $\mathrm{x}$ & $\mathrm{x}$ & - \\
\hline 8 & 659571 & 8791205 & 154,0 & - & $\mathrm{x}$ & - & - & $\mathrm{x}$ & $\mathrm{x}$ & - \\
\hline 9 & 659648 & 8791120 & 153,0 & $\mathrm{x}$ & - & - & - & $\mathrm{x}$ & $\mathrm{x}$ & - \\
\hline 10 & 659835 & 8791095 & 158,0 & $\mathrm{x}$ & - & - & - & $\mathrm{x}$ & $\mathrm{x}$ & - \\
\hline 11 & 658980 & 8792465 & 150,0 & $\mathrm{x}$ & - & - & - & $\mathrm{x}$ & $\mathrm{x}$ & - \\
\hline 12 & 659263 & 8792385 & 156,5 & $\mathrm{x}$ & - & - & - & $\mathrm{x}$ & - & $\mathrm{x}$ \\
\hline 13 & 657346 & 8792770 & 168,0 & $\mathrm{x}$ & - & - & - & $\mathrm{x}$ & - & $\mathrm{x}$ \\
\hline 14 & 657096 & 8792856 & 175,0 & $\mathrm{x}$ & - & - & - & $\mathrm{x}$ & - & $\mathrm{x}$ \\
\hline 15 & 657023 & 8792878 & 171,0 & $\mathrm{x}$ & - & - & - & $\mathrm{x}$ & - & $\mathrm{x}$ \\
\hline 16 & 657319 & 8792967 & 172,0 & $\mathrm{x}$ & - & - & - & $\mathrm{x}$ & - & $\mathrm{x}$ \\
\hline 17 & 657270 & 8792928 & 160,7 & $\mathrm{x}$ & - & - & - & $\mathrm{x}$ & $\mathrm{x}$ & - \\
\hline 18 & 657620 & 8793264 & 165,8 & $\mathrm{x}$ & - & - & - & $\mathrm{x}$ & $\mathrm{x}$ & - \\
\hline 19 & 657629 & 8793235 & 162.4 & $\mathrm{x}$ & - & - & $\mathrm{x}$ & - & $\mathrm{x}$ & - \\
\hline 20 & 657675 & 8793251 & 164,0 & $\mathrm{x}$ & - & - & $\mathrm{x}$ & - & $\mathrm{x}$ & - \\
\hline 21 & 659797 & 8793317 & 160,0 & $\mathrm{x}$ & - & - & $\mathrm{x}$ & - & $\mathrm{x}$ & - \\
\hline 22 & 659083 & 8793709 & 158,0 & $\mathrm{x}$ & - & $\mathrm{x}$ & - & - & $\mathrm{x}$ & - \\
\hline 23 & 659656 & 8792858 & 159,0 & $\mathrm{x}$ & - & - & $\mathrm{X}$ & - & $\mathrm{x}$ & - \\
\hline 24 & 658830 & 8792252 & 133,0 & - & $\mathrm{x}$ & - & - & $\mathrm{x}$ & - & $\mathrm{x}$ \\
\hline 25 & 659657 & 8788083 & 175,0 & - & $\mathrm{x}$ & - & $\mathrm{x}$ & - & $\mathrm{x}$ & - \\
\hline 26 & 658360 & 8788038 & 150,0 & $\mathrm{x}$ & - & - & - & $\mathrm{X}$ & $\mathrm{x}$ & - \\
\hline Total & - & - & - & 22 & 4 & 1 & 6 & 19 & 20 & 6 \\
\hline
\end{tabular}

Nota-se que mais de $90 \%$ das nascentes encontram-se altamente comprometidas, principalmente por estarem em maior número degradadas. Com isso, sem proteção da cobertura vegetal, tornam-se sujeitas a interromper (a médio e em longo prazo), a produção de água.

Leal et al. (2017) enfatizam que a ocupação antrópica em áreas como essas de preservação permanente destinadas à vegetação nativa, pode alterar o ciclo hidrológico e a regeneração natural no sub-bosque. Ainda segundo os autores, a ausência da proteção ao redor das nascentes também pode facilitar o fluxo de pessoas e animais, ocasionando o pisoteio e por consequência, a alteração na vazão das nascentes.

$\mathrm{Na}$ possibilidade necessária da recuperação dessas áreas, afim da restauração ecológica da vegetação nativa, conservação e proteção das nascentes, autores como Souza et al. (2012),
Alvarenga et al. (2016) e Rodrigues et al. (2020), destacam a aplicabilidade de algumas estratégias que podem ser empregadas, a depender do nível de perturbação das áreas de nascentes, como a condução da regeneração natural por isolamento da área; o enriquecimento da diversidade genética com a introdução de indivíduos de espécies já existentes no local, como facilitadoras do processo de regeneração; utilização de poleiros artificiais a favorecer a entrada de sementes, promoção da dispersão e atração de dispersores de sementes, bem como o plantio direto de mudas ou semeadura direta.

Quanto ao tipo de recarga, o maior número de nascentes foi classificado como pontual (22), representando $84,61 \%$ e apenas quatro $(15,39 \%)$ como difusa. Esta condição pode ser justificada, pois as nascentes encontram-se situadas na parte mais alta desta unidade de planejamento, com altitude variando de $133 \mathrm{~m}$ a $175 \mathrm{~m}$. Em área mais 
declivosas, comumente espera-se que as nascentes difusas sejam predominantes (Pinto et al. 2004, 2012), fato comprovado neste trabalho.

Analisando o estado de conservação das nascentes e comparando-os com os resultados encontrados por Pinto et al. (2005b), em estudo realizado em nascentes da Bacia Hidrográfica do Ribeirão Santa Cruz em Lavras-MG, que registraram um número de nascentes perturbadas maior do que as do tipo degradadas, percebe-se que no alto curso do rio Piauitinga, as nascentes apresentam-se em maior número degradadas do que perturbadas, caracterizando-se, assim, um avançado estágio de degradação ambiental.

Esta condição degradante, torna-se preocupante, uma vez que se trata das principais nascentes neste município, responsáveis pela maior vazão nesta região do rio Piauitinga. Em consequência, podendo vir a comprometer o abastecimento de água em volume e principalmente em sua qualidade, diferentemente do que se pode esperar de nascentes completamente preservadas, conforme Marmontel e Rodrigues (2015).

Em trabalho também realizado no estado de Sergipe, na Sub-Bacia Hidrográfica do rio Poxim, Ferreira et al. (2011) diagnosticaram as suas 20 principais nascentes e identificaram que apenas duas estavam preservadas e 13 encontravam-se degradadas. Segundo os referidos autores, as principais formas de antropização, com consequentes desmatamentos, são principalmente para uso na forma de lazer e para fins de produção agrícola $(50 \%)$ e pastoril $(35 \%)$.

Resende et al. (2009), estudando o estado de conservação de 70 nascentes da Bacia Hidrográfica do Córrego-Feio em Patrocínio-MG, constataram $63 \%$ das nascentes em estado de perturbadas, $20 \%$ classificadas como preservadas e $17 \%$ como degradadas. Os referidos autores ainda observaram como principais fatores de perturbação, os acessos de bovinos nas nascentes, a presença de plantas invasoras e o manejo inadequado dos solos no entorno destas.

Das 39 nascentes avaliadas por Pieroni et al. (2019) em Microbacias Hidrográficas do Córrego Ibitinga, Rio Claro-SP, $72 \%$ encontravam-se altamente degradadas com $97,4 \%$ das nascentes circundadas por áreas ocupadas com pastagens, cana-de-açúcar e silvicultura. Ainda segundo estes autores, a proximidade com estradas e a ausência de proteção, também foram fatores de impactos encontrados com maior frequência.

Situações essas que corroboram, portanto, com uma alta taxa de nascentes degradadas por conta de usos e ocupação do solo de forma comum observada também nas nascentes do rio Piauitinga, em seu alto curso, onde se encontram ocupadas principalmente por pastagens $(50 \%)$ e agricultura $(38 \%)$. Os outros usos observados nas nascentes com alto grau de degradação são construções de residências e pequenos barramentos para lavagem de roupas, além de extração de areia para usos diversos. Nota-se ainda que $58 \%$ das nascentes têm mais de um tipo de uso e ocupação do solo.

Observou-se que das nascentes, uma $(3,8 \%)$ é em área preservada e outra (3,8\%) em área urbanizada. E que cinco $(19,2 \%)$ encontra-se em área de regeneração, quatro $(15,4 \%)$ com barragem para lavanderia e, duas nascentes $(7,8 \%)$ para a extração de areia.

Quando analisados os usos e ocupação dos solos das áreas das nascentes e, comparando-os aos resultados obtidos por Santos (2009) nos municípios de Salgado e Boquim na mesma Sub-Bacia Hidrográfica do Piauitinga, percebe-se que as áreas de nascentes desses três municípios possuem as suas classes de maior uso e ocupação dos solos destinadas à lavoura permanente e pecuária (pastagem).

Os resultados observados nessa unidade de planejamento também são semelhantes ao que foi encontrado por Ferreira et al. (2011) no rio Poxim em Sergipe em que, das 20 principais nascentes diagnosticadas, 13 nascentes $(65 \%)$ encontravam-se em estado de conservação degradadas. Seguidas por $25 \%$ em perturbadas, com predomínio da agricultura e das pastagens como principais usos, associado aos usos domésticos e para fins de lazer.

Ao confrontar os tipos de ocupação e uso do solo obtidos nas nascentes do presente estudo com os resultados encontrados por Valle Júnior et al. (2010) na Bacia Hidrográfica do rio Tijuco, Ituiutaba-MG e aos obtidos por Pinto et al. (2005b) na Bacia Hidrográfica do Ribeirão Santa Cruz, Lavras-MG, observa-se que os solos são destinados também em sua maioria à pecuária (pastagem) em todos os estudos citados anteriormente com valores de $50 \%, 57,02 \%$ e $62,41 \%$, respectivamente.

Quanto ao fluxo de água, 20 nascentes apresentaram-se como perenes e seis como intermitentes, conforme pode ser visto ainda na Tabela 1. Este fato acontece devido ao recapeamento do Grupo Barreiras ser pouco profundo nessa região, tendo, consequentemente, um lençol freático raso e abundante segundo Magalhães et al. (2012).

A presença de nascentes intermitentes pode estar associada, provavelmente, ao seu estado de degradação. Os desmatamentos realizados para uso alternativo do solo podem ter contribuído para a condição de intermitência, pois algumas das nascentes passam a ter vazão somente no período chuvoso. Ocorrendo em função do deslocamento do fluxo de água, observado em algumas delas, posteriormente a este período e no estabelecimento da estação seca na região. Podendo ocasionar a migração dos olhos d'agua para outros locais e causar a intermitência dos mananciais, segundo Souza et al. (2019), além de refletir na quantidade e na qualidade de água de suas nascentes.

Quanto ao aspecto florístico, foram encontradas 76 espécies (sendo oito não 


\section{Ferreira et al.}

identificadas) de 58 gêneros distribuídos em 36 famílias botânicas, sendo possível classificar 28 espécies como pioneiras, 21 espécies como clímax exigentes de luz e cinco espécies como clímax tolerantes à sombra. Por outro lado, 21 espécies permanecem sem a devida classificação, uma vez que não foram encontrados registros quanto a esta informação nas literaturas consultadas (Tabela 2).

Tabela 2. Relação das famílias botânicas e espécies arbóreo-arbustivas ocorrentes na vegetação de entorno de nascentes da Sub-Bacia Hidrográfica do rio Piauitinga, município de Lagarto-SE com os respectivos nomes populares. Em que: G.E. - grupo ecológico; P - pioneira; CL - clímax exigente em luz; CS - clímax tolerante à sombra e NC - não-classificado.

\begin{tabular}{|c|c|c|c|c|}
\hline Família Botânica & Nome Científico & Nome Popular & GE & Registro \\
\hline \multirow{7}{*}{ Anacardiaceae } & Anacardium giganteum W.Hancock ex Engl. & cajuí & $\mathrm{CL}$ & ASE/10481 \\
\hline & Anacardium occidentale $\mathrm{L}$. & cajueiro-verdade & $\mathrm{CL}$ & ASE/10453 \\
\hline & Anacardium sp. & cajueiro-bravo & $\mathrm{NC}$ & - \\
\hline & Astronium urundeuva (M.Allemão) Aubl. & aroeira-do-sertão & $\mathrm{CL}$ & - \\
\hline & Mangifera indica $\mathrm{L}$. & mangueira & $\mathrm{CL}$ & MAC/47912 \\
\hline & Tapirira guianensis Aubl. & pau-pombo & $\mathrm{P}$ & MAC/47914 \\
\hline & Xylopia frutescens Aubl. & pindaíba & $\mathrm{P}$ & ASE/10440 \\
\hline Apocynaceae & Himatanthus obovatus (Müll. Arg.) Woodson & cajueiro-da-velha & $\mathrm{CS}$ & ASE/14910 \\
\hline \multirow{2}{*}{ Araliaceae } & Didymopanax morototoni (Aubl.) Decne. \& Planch. & pé-de-galinha & $\mathrm{C}$ & ASE/10482 \\
\hline & Didymopanax macrocarpus (Cham. \& Schltdl.) Seem. & pé-de-galinha & $\mathrm{P}$ & - \\
\hline Asteraceae & Moquiniastrum oligocephalum (Gardner) G.Sancho & candeia & $\mathrm{P}$ & - \\
\hline Bignoniaceae & Tabebuia stenocalyx Sprague \& Stapf & folha-larga & $\mathrm{NC}$ & MAC/35347 \\
\hline Burseraceae & Protium heptaphyllum (Aubl.) Marchand & amescla & $\mathrm{CS}$ & ASE/10456 \\
\hline Cannabaceae & Trema micrantha (L.) Blume & trema & $\mathrm{P}$ & MAC/47764 \\
\hline \multirow{2}{*}{ Chrysobalanaceae } & Hirtella racemosa Lam. & bula-cinza & $\mathrm{NC}$ & ASE/10448 \\
\hline & Moquilea tomentosa Benth. & oiti & $\mathrm{P}$ & - \\
\hline Calophyllaceae & Kielmeyera sp. & pé-de-veado & $\mathrm{P}$ & - \\
\hline Clusiaceae & Symphonia globulifera L.f. & pau-sangue & $\mathrm{NC}$ & - \\
\hline Dilleniaceae & Curatella americana $\mathrm{L}$. & lixeira & $\mathrm{P}$ & ASE/10457 \\
\hline \multirow[t]{7}{*}{ Euphorbiaceae } & Croton urucurana Baill. & sebastiana & $\mathrm{P}$ & - \\
\hline & $\begin{array}{l}\text { Abarema cochliacarpos (Gomes) Barneby \& } \\
\text { J.W.Grimes }\end{array}$ & $\begin{array}{l}\text { contas-de-nossa- } \\
\text { senhora }\end{array}$ & $\mathrm{NC}$ & ASE/10443 \\
\hline & Andira fraxinifolia Benth. & angelim & $\mathrm{CS}$ & ASE/10483 \\
\hline & Bowdichia virgilioides Kunth & sucupira & $\mathrm{CL}$ & ASE/10471 \\
\hline & Cassia grandis L.f. & canafístula & $\mathrm{CS}$ & MAC/47920 \\
\hline & Clitoria fairchildiana R.A.Howard & sombreiro & $\mathrm{NC}$ & - \\
\hline & Inga laurina (Sw.) Willd. & ingá & $\mathrm{CL}$ & ASE/10454 \\
\hline \multirow[t]{7}{*}{ Fabaceae } & Machaerium aculeatum Raddi & mau-vizinho & $\mathrm{P}$ & MAC/47919 \\
\hline & Mimosa tenuiflora (Willd.) Poir. & jurema & $\mathrm{P}$ & MAC/47870 \\
\hline & $\begin{array}{l}\text { Senna macranthera (DC. ex Collad.) H.S.Irwin \& } \\
\text { Barneby }\end{array}$ & flor-de-são-joão & $\mathrm{P}$ & $\mathrm{ASE} / 10450$ \\
\hline & Senna spectabilis (DC.) H.S.Irwin \& Barneby & cássia & $\mathrm{P}$ & - \\
\hline & Stryphnodendron pulcherrimum (Willd.) Hochr. & maria-farinha & $\mathrm{CL}$ & ASE/10455 \\
\hline & Swartzia apetala Raddi & olho-de-galo & $\mathrm{CL}$ & MAC/47789 \\
\hline & Tachigali densiflora (Benth.) L.G.Silva \& H.C.Lima & pau-fava & $\mathrm{CL}$ & MAC/35357 \\
\hline Hypericaceae & Vismia guianensis (Aubl.) Choisy & batom & $\mathrm{P}$ & ASE/10452 \\
\hline Lamiaceae & Aegiphila integrifolia (Jacq.) Moldenke & bordão-de-velho & $\mathrm{P}$ & ASE/10451 \\
\hline Lauraceae & Ocotea glomerata (Nees) Mez & louro & $\mathrm{CL}$ & MAC/47791 \\
\hline \multirow{3}{*}{ Lecythidaceae } & Eschweilera ovata (Cambess.) Mart. ex Miers & biriba & $\mathrm{CL}$ & MAC/47774 \\
\hline & Gustavia augusta L. & sapucaia & $\mathrm{CS}$ & - \\
\hline & Byrsonima sericea DC. & murici & $\mathrm{P}$ & ASE/10629 \\
\hline \multirow[t]{2}{*}{ Malpighiaceae } & Byrsonima sp. & guarama & $\mathrm{P}$ & - \\
\hline & Byrsonima verbascifolia (L.) DC. & murici & $\mathrm{P}$ & ASE/10438 \\
\hline Malvaceae & Guazuma ulmifolia Lam. & mutamba & $\mathrm{P}$ & MAC/47825 \\
\hline \multirow{2}{*}{ Melastomataceae } & Pleroma mutabile (Vell.) Triana & flor-de-natal & $\mathrm{P}$ & ASE/14902 \\
\hline & Tibouchina sp. & quaresmeira & $\mathrm{P}$ & - \\
\hline \multirow[t]{2}{*}{ Moraceae } & Artocarpus heterophyllus Lam. & jaqueira-verdadeira & $\mathrm{P}$ & ASE/10478 \\
\hline & Campomanesia xanthocarpa (Mart.) O. Berg & guabiroba & $\mathrm{CL}$ & ASE/14933 \\
\hline \multirow{3}{*}{ Myrtaceae } & Myrcia pseudopauciflora A.R.Lourenço \& E.Lucas & vara-branca & $\mathrm{NC}$ & MAC/47819 \\
\hline & Myrcia sp. & canela-de-veado & $\mathrm{NC}$ & MAC/47808 \\
\hline & Myrcia obovata (O.Berg) Nied. & murta-branca & $\mathrm{NC}$ & ASE/10446 \\
\hline
\end{tabular}




\begin{tabular}{|c|c|c|c|c|}
\hline & Psidium guajava $\mathrm{L}$. & goiabeira & $\mathrm{CL}$ & MAC/47805 \\
\hline & Syzygium jambolanum (Lam.) DC. & jamelão & CL & MAC/47843 \\
\hline & Syzygium malaccense (L.) Merr. \& L.M.Perry & jambo & CL & - \\
\hline \multirow[b]{2}{*}{ Nyctaginaceae } & Pisonia sp. 1 & bandola & $\mathrm{NC}$ & - \\
\hline & Pisonia sp. 2 & joão-mole & $\mathrm{NC}$ & MAC/47833 \\
\hline Ochnaceae & Ouratea sp. & ouratea & $\mathrm{CL}$ & ASE/10458 \\
\hline Phyllanthaceae & Richeria grandis Vahl & jaqueira-brava & $\mathrm{NC}$ & ASE/10447 \\
\hline Primulaceae & Myrsine guianensis (Aubl.) Kuntze & tapiroroca & $\mathrm{P}$ & MAC/35346 \\
\hline Proteaceae & Roupala montana Aubl. & carne-de-vaca & $\mathrm{CL}$ & MAC/47792 \\
\hline Rubiaceae & Genipa americana $\mathrm{L}$. & jenipapo & $\mathrm{CL}$ & ASE/10465 \\
\hline Rutaceae & Zanthoxylum rhoifolium Lam. & laranjeira-brava & $\mathrm{P}$ & ASE/10441 \\
\hline \multirow{2}{*}{ Sapindaceae } & Allophylus edulis (A.St.-Hil. et al) Hieron. ex Niederl. & estralador & $\mathrm{CL}$ & - \\
\hline & Cupania vernalis Cambess. & camboatá & $\mathrm{CL}$ & - \\
\hline Salicaceae & Casearia sylvestris $\mathrm{Sw}$. & ganhador & $P$ & MAC/35355 \\
\hline Sapotaceae & Manilkara sp. & maçaranduba & $\mathrm{NC}$ & $\mathrm{ASE} / 10449$ \\
\hline Simaroubaceae & Simarouba amara Aubl. & pau-paraíba & $\mathrm{CL}$ & ASE/10461 \\
\hline Solanaceae & Cestrum axillare Vell. & quarana & $\mathrm{P}$ & ASE/10437 \\
\hline Urticaceae & Cecropia pachystachya Trécul & embaúba & $P$ & ASE/10442 \\
\hline Vochysiaceae & Vochysia thyrsoidea Pohl & volquízia & $\mathrm{P}$ & MAC/35358 \\
\hline Morfoespécie 1 & - & - & $\mathrm{NC}$ & - \\
\hline Morfoespécie 2 & - & - & $\mathrm{NC}$ & - \\
\hline Morfoespécie 3 & - & - & $\mathrm{NC}$ & - \\
\hline Morfoespécie 4 & - & - & $\mathrm{NC}$ & - \\
\hline Morfoespécie 5 & - & - & $\mathrm{NC}$ & - \\
\hline Morfoespécie 6 & - & - & $\mathrm{NC}$ & - \\
\hline Morfoespécie 7 & - & - & $\mathrm{NC}$ & - \\
\hline Morfoespécie 8 & - & - & $\mathrm{NC}$ & - \\
\hline
\end{tabular}

Observa-se que dentre os grupos ecológicos (categorias sucessionais) identificados, mais da metade $(50,9 \%)$ das espécies amostradas reconhecidas são espécies pioneiras. Predominância essa, contribuída, possivelmente, em decorrência da interferência antrópica nas áreas das nascentes, retardando o estabelecimento das espécies clímax.

Por outro lado, no processo da restauração florestal a dominância dessas espécies pioneiras é importante por favorecer a regeneração natural em resposta à degradação, promovendo o sombreamento necessário para o crescimento das outras espécies e também por propiciar uma melhor cobertura do terreno, essencial para a proteção dos solos. Importante ainda, conforme Ferreira et al. (2013), pela interação com a fauna, auxiliando o restabelecimento da dinâmica e do equilíbrio dos ecossistemas.

Dentre a riqueza de espécies, a famílias Fabaceae (13) seguida por Myrtaceae (7) e Anacardiaceae (7), foram as mais expressivas. Dos gêneros, os mais ricos foram Anacardium (Anacardiaceae), Myrcia (Myrtaceae) e Byrsonima (Malpighiaceae) com três espécies cada. As famílias mais abundantes foram: Anacardiaceae $(24,84 \%)$, Fabaceae (14,64\%), Urticaceae $(12,45 \%)$, Malpighiaceae $(7,81 \%)$, Simaroubaceae $(7,35 \%)$ e Dilleniaceae $(7,16 \%)$, totalizando $79,42 \%$ dos indivíduos amostrados.

Observa-se que as famílias Urticaceae, Simaroubaceae e Dilleniaceae representaram grande abundância com apenas uma espécie cada.
Diferentemente da espécie Myrtaceae que com sete espécies respondeu apenas com 2,52\% em abundância. Situação que pode ser explicada, provavelmente, pela diferença de desenvolvimento e ocupação espacial entre as espécies, relacionadas ao grupo de sucessão ecológica as quais pertencem. A exemplo das espécies representativas de cada uma das famílias Urticaceae $(C$. pachystachya) e Dilleniaceae $(C$. americana $)$ encontrada nas nascentes que pertencem ao grupo das pioneiras. Diferentemente da grande parte das espécies encontradas das Myrtaceae que são clímax.

Além do mais, essa situação pode também estar relacionada a possíveis interferências antrópicas na área que, por razões de redução da vegetação por desmatamentos, tende a descaracterizar a composição florística original das nascentes, por exemplo.

Em um estudo sobre a composição florística e caracterização do estado de conservação de nascentes no Centro-Leste da Bacia Hidrográfica do rio Itapicuru, Semiárido da Bahia, Jesus et al. (2018) observaram maior abundância nas espécies catingueira (Poincianella pyramidalis (Tul.) L.P.Queiroz) com 23,7\% dos indivíduos seguida por ingá (Inga sp.) com 13,9\% dos indivíduos presentes nas nascentes. Fabaceae foi à família botânica com maior riqueza (44\%), evidenciando a presença dessa família botânica nesses ambientes.

Foi observado ainda no presente estudo uma ocorrência acentuada de espécies exóticas correspondendo a $38,8 \%$ do total, a exemplo de 
mangueira (Mangifera indica L. - Anacardiaceae), jamelão (Syzygium jambolanum (Lam.) DC. Myrtaceae) e jaqueiras (Artocarpus heterophyllus Lam. - Moraceae), além de coqueiros (Cocos nucifera L. - Arecaceae).

A introdução dessas espécies nesses ambientes não é desejável em virtude dos problemas que provocam, sobretudo interferindo nos processos ecológicos, desequilibrando as relações bióticas naturais, deteriorando a diversidade e modificando a paisagem original.

Ressalta-se que os levantamentos florísticos do estrato arbustivo-arbóreo foram realizados em 25 nascentes, do total de 26 diagnosticadas, pois em uma não havia indivíduos arbustivo-arbóreos nativos, apenas havia a presença de 25 coqueiros ( $C$. nucifera). Assim, ela não foi considerada para efeito de análises florística e fitossociológica. Desse modo, foram registrados 1.550 indivíduos, distribuídos em 76 espécies, sendo oito não identificados (morfoespécies). A área basal correspondente foi de 68,46 $\mathrm{m}^{2} \cdot \mathrm{ha}^{-1} \mathrm{e}$ a densidade média obtida foi de 98,73 ind.ha' ${ }^{-1}$ (Tabela 3 ).

Tabela 3. Estimativa dos parâmetros fitossociológicos de nascentes estudadas na Sub-Bacia Hidrográfica do rio Piauitinga, município de Lagarto-SE. Em que: $\mathrm{Ni}$ - número de indivíduos; $\mathrm{AB}$ - área basal ( $\left.\mathrm{m}^{2}\right)$; DA - densidade absoluta (ind.ha-1); DR - densidade relativa (\%); FA - frequência absoluta (\%); FR - frequência relativa (\%); DoA - dominância absoluta $\left(\mathrm{m}^{2} \cdot \mathrm{ha}^{-1}\right)$; DoR - dominância relativa (\%); VI - valor de importância.

\begin{tabular}{|c|c|c|c|c|c|c|c|c|c|}
\hline Espécie & $\mathbf{N i}$ & $\mathbf{A B}$ & DA & DR & FA & FR & DoA & DoR & VI \\
\hline Tapirira guianensis Aubl. & 306 & 14,874 & 19,49 & 19,69 & 60,0 & 4,92 & 0,947 & 21,72 & 46,334 \\
\hline Cecropia pachystachya Trécul & 193 & 10,074 & 12,293 & 12,42 & 70,0 & 5,74 & 0,642 & 14,71 & 32,871 \\
\hline Inga laurina (Sw.) Willd. & 128 & 7,757 & 8,153 & 8,24 & 30,0 & 2,46 &, 494 & 11,33 & 2,026 \\
\hline Byrsonima sericea DC. & 117 & 2,259 & 7,452 & 7,53 & 70,0 & 5,74 & 0,144 & 3,3 & 16,567 \\
\hline Simarouba amara Aubl. & 114 & 8,437 & 7,261 & 7,34 & 5,0 & 0,41 & 0,537 & 12,32 & 20,068 \\
\hline Curatella americana $\mathrm{L}$. & 111 & 1,951 & 7,07 & 7,14 & 50,0 & 4,1 & 0,124 & 2,85 & 14,091 \\
\hline Symphonia globulifera $\mathrm{L}$ & 52 & 1,426 & 3,312 & 3,35 & 20,0 & 1,64 & 0,091 & 2,08 & 7,068 \\
\hline Richeria grandis Vahl & 40 & 2,909 & 2,548 & 2,57 & 20,0 & 1,64 & 0,185 & 4,25 & 8,463 \\
\hline Casearia sylvestris $\mathrm{Sw}$. & 28 & 1,052 & 1,783 & 1,8 & 35,0 & 2,87 &, 067 & 1,54 & 6,207 \\
\hline Anacardium occidentale $\mathrm{L}$. & 27 & 2,547 & 1,72 & 1,74 & 30,0 & 2,46 & 0,162 & 3,72 & 7,917 \\
\hline Protium heptaphyllum (Aubl.) N & 27 & 1,056 & 1,72 & 1,74 & 15,0 & 1,23 &, 067 & 1,54 & 4,51 \\
\hline Bowdichia virgilioides $\mathrm{K}$ & 23 & 0,397 & 1,465 & 1,48 & 50,0 & 4,1 &, 025 & 0,58 & 6,158 \\
\hline Hirtella rac & 22 & 0,221 & 1,401 & 1,42 & 30,0 & 2,46 & 0,014 & 0,32 & 4,198 \\
\hline Mangifer & 21 & 4,02 & 1,338 & 1,35 & 30,0 & 2,46 & 0,256 & 5,87 & 9,682 \\
\hline Tabebuia ster & 21 & 0,623 & 1,338 & 1,35 & 20,0 & 1,64 & 0,04 & 0,91 & 3,901 \\
\hline Himatanthus obovatus (Müll. Arg.) Woodson & 19 & 0,32 & 1,21 & 1,22 & 35,0 & 2,87 & 0,02 & 0,47 & 4,559 \\
\hline Clitoria fairchildiana $\mathrm{R}$ & 19 & 1,088 & 1,21 & 1,22 & 5,0 & 0,41 & 0,069 & 1,59 & 3,222 \\
\hline Aegiphila in & 17 & 0,224 & 1,083 & 1,09 & 25,0 & 2,05 & 14 & 0,33 & 3,471 \\
\hline Anacardium & 14 & 0,506 & 0,892 & 0,9 & 25,0 & 2,05 & 0,032 & 0,74 & 3,688 \\
\hline Myrcia obov & 13 & 0,149 & 0,828 & 0,84 & 20,0 & 1,64 & 0,01 & 0,22 & 2,694 \\
\hline Andira & 1 & 26 & 0,828 & 0,84 & 30,0 & 2,46 & 0,021 & 0,48 & 3,772 \\
\hline Zanthos & 12 & 0,101 & 0,764 & 0,77 & 20,0 & 1,64 &, 006 & 0,15 & 2,56 \\
\hline $\begin{array}{l}\text { Moquiniastrum oligocephalum (G } \\
\text { G.Sancho }\end{array}$ & 12 & 0,089 & 0,764 & 0,77 & 25,0 & 2,05 & 0,006 & 0,13 & 2,951 \\
\hline rutescens Aubl. & 11 & 0,271 & 0,701 & 0,71 & 15,0 & 1,23 &, 017 & 0,4 & 2,333 \\
\hline anum $(\mathrm{L}$ & 10 & 0,124 & 0,637 & 0,64 & 10,0 & 0,82 & 0,008 & 0,18 & 1,644 \\
\hline Stryphnodendron pulcherr & 10 & 0,654 & 0,637 & 0,64 & 25,0 & 2,05 & 0,042 & 0,96 & 3,648 \\
\hline Pisonia sp. 1 & 9 & 0,071 & 0,573 & 0,58 & 15,0 & 1,23 & 0,005 & 0,1 & 1,913 \\
\hline Swartzia apeto & 8 & 0,151 & & 0,51 & 30,0 & 2,46 & 0,01 & 0,22 & 3,194 \\
\hline $\begin{array}{l}\text { Abarema cochliacarpos (Gomes) Barneby \& } \\
\text { J.W.Grimes }\end{array}$ & 8 & 0,099 & 0,51 & 0,51 & 15,0 & 1,23 & 0,006 & 0,14 & 1,889 \\
\hline Eschweilera ovata (Cambess.) Mart. ex Miers & 8 & 0,10 & 0,51 & 0,51 & 10,0 & 0,82 & 0,011 & 0,26 & 1,597 \\
\hline Ouratea sp. & 7 & 0,049 & 0,446 & 0,45 & 10,0 & 0,82 & 0,003 & 0,07 & 1,341 \\
\hline Campomane & 7 & 0,077 & 0,446 & 0,45 & 10,0 & 0,82 & 0,005 & 0,11 & 1,382 \\
\hline rantha (L.) Blume & 7 & 0,211 & 0,446 & 0,45 & 5,0 & 0,41 & 0,013 & 0,31 & 1,169 \\
\hline Pisonia & 6 & 0,048 & 0,382 & 0,39 & 10,0 & 0,82 & 0,003 & 0,07 & 1,276 \\
\hline Mort & 6 & 0,046 & 0,382 & 0,39 & 10,0 & 0,82 & 0,003 & 0,07 & 1,273 \\
\hline Anacardium sp. & 5 & 0,057 & 0,318 & 0,32 & 15,0 & 1,23 & 0,004 & 0,08 & 1,634 \\
\hline Tachigali densiflora (Benth.) L.G.Silva \& & 5 & 1,381 & 0,318 & 0,32 & 5,0 & 0,41 & 0,088 & 2,02 & 2,749 \\
\hline Morfoespécie 5 & 5 & 0,069 & 0,318 & 0,32 & 10,0 & 0,82 & 0,004 & 0,1 & 1,242 \\
\hline Artocarpus heterophyllus Lam. & 4 & 0,392 & 0,255 & 0,26 & 15,0 & 1,23 & 0,025 & 0,57 & 2,06 \\
\hline
\end{tabular}




\begin{tabular}{|c|c|c|c|c|c|c|c|c|c|}
\hline Vismia guianensis (Aubl.) Choisy & 4 & 0,052 & 0,255 & 0,26 & 15,0 & 1,23 & 0,003 & 0,08 & 1,563 \\
\hline Manilkara sp. & 4 & 0,036 & 0,255 & 0,26 & 15,0 & 1,23 & 0,002 & 0,05 & 1,54 \\
\hline Mimosa tenuiflora (Willd.) Poir. & 4 & 0,337 & 0,255 & 0,26 & 15,0 & 1,23 & 0,021 & 0,49 & 1,979 \\
\hline Pleroma mutabile (Vell.) Triana & 4 & 0,043 & 0,255 & 0,26 & 10,0 & 0,82 & 0,003 & 0,06 & 1,1 \\
\hline Morfoespécie 4 & 4 & 0,084 & 0,255 & 0,26 & 15,0 & 1,23 & 0,005 & 0,12 & 1,6 \\
\hline Psidium guajava $\mathrm{L}$. & 4 & 0,036 & 0,255 & 0,26 & 10,0 & 0,82 & 0,002 & 0,05 & 1,12 \\
\hline Morfoespécie 2 & 4 & 0,038 & 0,255 & 0,26 & 5,0 & 0,41 & 0,002 & 0,06 & 0,72 \\
\hline Tibouchina sp. & 4 & 0,025 & 0,255 & 0,26 & 10,0 & 0,82 & 0,002 & 0,04 & 1,11 \\
\hline Byrsonima sp. & 3 & 0,019 & 0,191 & 0,19 & 10,0 & 0,82 & 0,001 & 0,03 & 1,0 \\
\hline $\begin{array}{l}\text { Didymopanax morototoni (Aubl.) Decne. \& } \\
\text { Planch. }\end{array}$ & 3 & 0,021 & 0,191 & 0,19 & 15,0 & 1,23 & 0,001 & 0,03 & 1,45 \\
\hline $\begin{array}{l}\text { Senna macranthera (DC. ex Collad.) } \\
\text { H.S.Irwin\&Barneby }\end{array}$ & 3 & 0,023 & 0,191 & 0,19 & 10,0 & 0,82 & 0,001 & 0,03 & 1,04 \\
\hline Roupala montana Aubl. & 3 & 0,027 & 0,191 & 0,19 & 10,0 & 0,82 & 0,002 & 0,04 & 1,05 \\
\hline Myrsine guianensis (Aubl.) Kuntze & 3 & 0,051 & 0,191 & 0,19 & 5,0 & 0,41 & 0,003 & 0,07 & 0,678 \\
\hline Ocotea glomerata (Nees) Mez & 3 & 0,092 & 0,191 & 0,19 & 5,0 & 0,41 & 0,006 & 0,13 & 0,737 \\
\hline Morfoes & 3 & 0,04 & 0,191 & 0,19 & 5,0 & 0,41 & 0,003 & 0,06 & 0,66 \\
\hline ectabilis (DC.) H.S.Irwin \&Bar & 3 & 0,029 & 0,191 & 0,19 & 5,0 & 0,41 & 0,002 & 0,04 & 0,64 \\
\hline Myrci & 2 & 0,059 & 0,127 & 0,13 & 5,0 & 0,41 & 0,004 & 0,09 & 0,625 \\
\hline Crotor & 2 & 0,222 & 0,127 & 0,13 & 10,0 & 0,82 & 0,014 & 0,32 & 1,27 \\
\hline Vochysia thyrsoidea Pohl & 2 & 0,158 & 0,127 & 0,13 & 10,0 & 0,82 & 0,01 & 0,23 & 1,1 \\
\hline Cupania v & 2 & 0,022 & 0,127 & 0,13 & 5,0 & 0,41 & 0,001 & 0,03 & 0,57 \\
\hline Cassi & 2 & 0,208 & 0,127 & 0,13 & 10,0 & 0,82 & 0,013 & 0,3 & 1,25 \\
\hline Morfoes & 2 & 0,01 & 0,127 & 0,13 & 5,0 & 0,41 & 0,001 & 0,01 & 0,55 \\
\hline Imifolia Lam. & 2 & 0,084 & 0,127 & 0,13 & 10,0 & 0,82 & 0,005 & 0,12 & 1,07 \\
\hline Syzygium malaccense (L.) Merr. \& L.M.Perry & 2 & 0,122 & 0,127 & 0,13 & 5,0 & 0,41 & 0,008 & 0,18 & 0,717 \\
\hline Cestrum axillare Vell. & 1 & 0,004 & 0,064 & 0,06 & 5,0 & 0,41 & 0 & 0,01 & 0,48 \\
\hline Astronium urundeuva (M.Allemão) Aubl. & 1 & 0,016 & 0,064 & 0,06 & 5,0 & 0,41 & 0,001 & 0,02 & 0,49 \\
\hline Kielmeyera sp. & 1 & 0,004 & 0,064 & 0,06 & 5,0 & 0,41 & 0 & 0,01 & 0,4 \\
\hline $\begin{array}{l}\text { Didymopanax macrocarpus (Cham. \& Schltdl.) } \\
\text { Seem. }\end{array}$ & 1 & 0,007 & 0,064 & 0,06 & 5,0 & 0,41 & 0 & 0,01 & 0,48 \\
\hline folia (L.) DC. & 1 & 0,005 & 0,064 & 0,06 & 5,0 & 0,41 & 0 & 0,01 & 0,48 \\
\hline Genipc & 1 & 0,07 & 0,064 & 0,06 & 5,0 & 0,41 & 0,004 & 0,1 & 0,57 \\
\hline Morfoe & 1 & 0,005 & 0,064 & 0,06 & 5,0 & 0,41 & 0 & 0,01 & 0,48 \\
\hline $\begin{array}{l}\text { Myrcia pseudopauciflora A.R.Lourenço \& } \\
\text { E.Lucas }\end{array}$ & 1 & 0,004 & 0,064 & 0,06 & 5,0 & 0,41 & 0 & 0,01 & 0 , \\
\hline Machaerium aculeatum Raddi & 1 & 0,077 & 0,064 & 0,06 & 5,0 & 0,41 & 0,005 & 0,11 & 0,58 \\
\hline $\begin{array}{l}\text { Allophylus edulis (A.St.-Hil. et al) Hieron. } \\
\text { exNiederl. }\end{array}$ & 1 & 0,007 & 0,064 & 0,06 & 5,0 & 0,41 & 0 & 0,01 & 0,48 \\
\hline Gustavia augusta $\mathrm{L}$. & 1 & 0,035 & 0,064 & 0,06 & 5,0 & 0,41 & 0,002 & 0,05 & 0,52 \\
\hline Morfoespécie 6 & 1 & 0,023 & 0,064 & 0,06 & 5,0 & 0,41 & 0,001 & 0,03 & 0,50 \\
\hline Moquilea tomentosa Benth. & 1 & 0,083 & 0,064 & 0,06 & 5,0 & 0,41 & 0,005 & 0,12 & 0,59 \\
\hline otal & 550 & 68,464 & 98,728 & 100 & 1215 & 100 & 4,353 & 100 & 30 \\
\hline
\end{tabular}

As dez espécies com maior densidade ( $T$. guianensis, C. pachystachya, I. laurina, B. sericea, $S$. amara, C. americana, S. globulifera, $R$. grandis, C. sylvestri e A. occidentale) representam $71,08 \%$ do número total de indivíduos.

Em relação à área basal, cinco espécies $(6,6 \%)$ contribuíram com $65,96 \%$ do total, a saber: T. guianensis contribuindo com $21,73 \%, C$. pachystachya (14,71\%), I. laurina (11,33\%), S. amara com $12,32 \%$ e $M$. indica com 5,87\%.

Percebe-se que pouco mais da metade da área basal revelada, está representada por apenas cinco espécies ocupando pouco mais da metade da área basal total, predominando, expressivamente, sobre as demais 71 espécies $(93,4 \%)$ restantes.
As espécies com maior frequência foram: $C$. pachystachya (70\%), B. sericea $(70 \%)$ e $T$. guianensis (60\%). Seguidas por $C$. americana e $B$. virgilioides ambas ocorrendo em $50 \%$ das áreas.

Em relação a dominância, 10 espécies tiveram maior representatividade ( $T$. guianensis, $C$. pachystachya, I. laurina, B. sericea, S. amara, C. americana, $S$. globulifera, $R$. grandis, $A$. occidentale e $M$. indica), totalizando $71,55 \%$ de todos os indivíduos amostrados. Situação essa que refletiu, diretamente, no valor de importância, onde essas mesmas espécies, embora em ordem diferente, $(T$. guianensis, C. pachystachya, I. laurina, B. sericea, $S$. amara, $C$. americana, $M$. indica, $R$. grandis, $A$. occidentale e $S$. globulifera) foram as que mais se 
destacaram, representando $71,6 \%$ do total de indivíduos.

$\mathrm{Na}$ análise fitossociológica realizada por Ferreira et al. (2007), na Reserva Ecológica de Dois Irmãos, Recife-PE, as espécies $T$. guianensis, $C$. pachystachya e $B$. sericea também apresentaram maior frequência sugerindo que estas espécies estão bem dispersas em ambiente ciliar em áreas de Mata Atlântica ou estendendo-se até às áreas de tensão ecológica.

Comparando-se as análises da vegetação arbóreo-arbustiva das nascentes da Sub-Bacia Hidrográfica do rio Piauitinga deste estudo com o realizado por Pinto et al. (2005b) na Bacia Hidrográfica do Ribeirão Santa Cruz, Lavras-MG, nota-se ainda que as espécies $C$. sylvestris, $C$. pachystachya $e T$. guianensis estão presentes em ambos os ambientes avaliados. Isto pode indicar a existência de uma ampla distribuição delas em toda a faixa costeira brasileira que sofre influência da Mata Atlântica original.

O valor de importância no presente estudo mostra uma frequência e dominância quase nula para treze espécies $(17,1 \%$ do total), as quais estão representadas por apenas um indivíduo cada. Nesse sentido, uma maior atenção para espécies nessas condições deve ser dada, principalmente pelo risco de sofrerem, dentre outros danos, a redução de sua variabilidade genética, por exemplo, o que torna comprometedor o estabelecimento dessas espécies na comunidade no longo prazo.

Por outro lado, também deve ser levada em consideração às espécies exóticas que foram encontradas nas áreas de nascentes, a fim de se evitar que se tornem invasoras, a exemplo de $M$. indica que foi observada como uma das dez espécies de maior valor de importância, ocorrendo em 30\% das nascentes, destacando-se em dominância e área basal.

Os índices de diversidade de ShannonWeaver (H') e de equabilidade de Pielou (J'), calculados para 25 nascentes foram, respectivamente, 3,04 nats.ind ${ }^{-1}$ e 0,71. De acordo com o índice de Shannon-Weaver (H'), há uma elevada diversidade, uma vez que os valores de H', na maioria das vezes, situam-se entre 1,3 e 3,5. Em relação ao índice de Pielou (J'), observa-se também uma elevada heterogeneidade florística nas nascentes estudadas. E a similaridade florística encontrada pelo coeficiente de Jaccard (SJ) nas 26 nascentes estudadas variou de $0,08 \%$ a $56,2 \%$. Notase ainda que as espécies estudadas nessas áreas do rio Piauitinga possuem uma razoável similaridade entre as nascentes e que são igualmente abundantes.

Comparando com outros trabalhos realizados em nascentes com condições parecidas, os índices situam-se com valores aproximados, como no estudo de Pinto et al. (2005c) que em 12 nascentes da Bacia Hidrográfica do Ribeirão Santa Cruz, Lavras-MG em uma Floresta Estacional Semidecidual Montana, encontraram 3,89 (H') e 0,72 (J') da vegetação analisada. Já no entorno de 14 nascentes no município de Salgado-SE, em uma Floresta Estacional Semidecidual Submontana, Oliveira et al. (2012) obtiveram 3,5 (H') e 0,76 (J') da vegetação analisada. Calazans et al. (2020) no município de Estância-SE, em Floresta Estacional-Savânica, observaram valores baixos de similaridade $(7,14 \%$ a $30,48 \%$ ) entre as nove nascentes.

\section{Conclusões}

$\mathrm{O}$ estudo permitiu compreender que as nascentes do alto curso da Sub-Bacia Hidrográfica do rio Piauitinga no município de Lagarto, Sergipe, com apenas uma classificada como preservada, apresentam-se degradadas com as áreas do entorno das nascentes utilizadas com finalidades diversas, destacando-se os usos agrícolas (temporários ou permanentes) e pecuários para fins de pastagens. Os remanescentes do entorno do maior número das nascentes encontram-se alteradas em sua estrutura, com as espécies atualmente presentes em maior parte de estádios iniciais de sucessão, em relação à vegetação original da nascente preservada.

Diante das circunstâncias encontradas, sugere-se a remoção do fator de degradação destas áreas, o isolamento adequado das nascentes e a posterior adoção de uma destas técnicas de restauração: a condução da regeneração natural da vegetação nativa das áreas perturbadas e o enriquecimento com mudas de espécies nativas por meio do plantio nas áreas degradadas.

\section{Agradecimentos}

Este trabalho foi realizado como parte do Projeto Adote um Manancial, financiado pela Secretaria de Meio Ambiente e dos Recursos Hídricos do Estado de Sergipe, realizado pelo Departamento de Ciências Florestais da Universidade Federal de Sergipe, Ministério Público Estadual de Sergipe/Comarca de Lagarto e Sociedade de Estudos Múltiplos e Artes - SEMEAR.

\section{Referências}

Aguiar Netto ADO, Magalhães LTS, Vasco AND, Gomes, JBV (2015) Indicadores ambientais do solo nas áreas de nascente da bacia hidrográfica do rio Piauitinga, Sergipe. Ambiência Guarapuava. 1(2): 457 - 472. doi: 10.5935/ambiencia.2015.02.13.

Alvarenga AP, Botelho SA, Pinheiro AC, Pereira IM (2016) Sobrevivência e crescimento inicial de espécies arbóreas nativas na restauração de nascentes no Sul de Minas Gerais. Enciclopédia Biosfera. 13(23): 1239-1250.

Alvares CA, Stape JL, Sentelhas PC, Gonçalves JLdeM, Sparovek G (2013) Mapa de classificação climática de Köppen para o Brasil. Zeitschrift 
Meteorologische. 22(6): 711-728. doi: 10.1127/0941-2948/2013/0507.

Bomfim EO, Gadelha CLM, Figueira HJA, Amorim JF, Amorim, DDS (2015) Sustentabilidade hidroambiental de nascentes na Bacia Hidrográfica do rio Gramame no Estado da Paraíba, Brasil. Sociedade \& Natureza. 27(3): 453-468. doi:10.1590/1982-451320150307.

Brasil (2012a) Lei no 12.651, de 25 de maio de 2012. Disponível em: $<$ http://www.planalto.gov.br/ccivil_03/ato20112014/2012/lei/112651.htm >. Acesso: 8 de maio de 2020.

Brasil (2012b) Manual Técnico da Vegetação Brasileira: Sistema Fitogeográfico, Inventário das Formações Florestais e Campestres, Técnicas e Manejo de Coleções Botânicas e Procedimentos para Mapeamentos. Instituto Brasileiro de Geografia e Estatística - IBGE. Rio de Janeiro-RJ, 271 p. Disponível em: https://biblioteca.ibge.gov.br/visualizacao/livros/liv 63011.pdf. Acesso: 25 de dezembro de 2020.

Brasil (2013) Manual Técnico de Uso da Terra. $3^{\circ}$ Edição. IBGE: Rio de Janeiro, 171p. Disponível em: $<$ https://biblioteca.ibge.gov.br/visualizacao/livr os/liv81615.pdf >. Acesso: 20 de julho de 2019.

Brasil (2020) Flora do Brasil 2020 em construção. Jardim Botânico do Rio de Janeiro. Disponível em: < http://floradobrasil.jbrj.gov.br/ >. Acesso: 02 de dezembro de 2020.

Brower JE, Zar JH (1984) Field and laboratory methods for general ecology. Dubuque: W.M.C. Brow. 226 p.

Calazans CC, Ferreira RA, Freire GDS, Pereira GS, Mann RS, Souza JL, Torres MFO, Nunes VV (2020) Caracterização de nascentes na Sub-Bacia Hidrográfica do Rio Piauitinga, município de Estância, Sergipe. Global Science And Technology, 13(1): 236-247.

Chaves ADCG, Santos RMDS, Santos JOD, Fernandes ADA, Maracajá PB (2013) A importância dos levantamentos florístico e fitossociológico para a conservação epreservação das florestas. Agropecuária Científica no Semiárido. Capina Grande, 9(2): 43-48. doi:10.30969/acsa.v9i2.449.

Cientec (2006) Mata Nativa 2: Sistema para análise fitossociológica e elaboração de planos de manejo de florestas nativas, 295p.
Climate-Data (2012) Dados climáticos mundiais Região de Lagarto, Sergipe, Brazil, 1982 - 2012. Disponível: $<$ https://pt.climate-data.org/america-do-

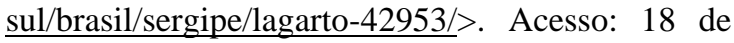
julho de 2019.

Coelho VHR, Montenegro SMGL, Almeida CN, Lima ERV, Ribeiro Neto A, Moura GSS (2014) Dinâmica do uso e ocupação do solo em uma Bacia Hidrográfica do semiárido brasileiro. Revista Brasileira de Engenharia Agrícola e Ambiental, 18(1): 64-72. doi: 10.1590/S141543662014000100009 .

Faria R, Botelho AS, Souza L (2012) Diagnóstico ambiental de áreas do entorno de 51 nascentes localizadas no município de Lavras, MG. Enciclopédia Biosfera. 8(15): 648-661.

Ferreira PI, Gomes JP, Batista F, Bernardi AP, Costa NCFD, Bortoluzzi RLDC, Mantovani A (2013) Espécies potenciais para recuperação de áreas de preservação permanente no Planalto Catarinense. Floresta e Ambiente, 20(2): 173-182. doi: 10.4322/floram.2013.003.

Ferreira RA, Aguiar Netto AO, Santos TIS, Santos BL, Matos EL (2011) Nascentes da Sub-Bacia Hidrográfica do Rio Poxim: da degradação à restauração. Revista Árvore. 35(2): 265-277. doi: 10.1590/S0100-67622011000200011.

Ferreira RLC, Marangon LC, Silva JAA, Rocha MS, Alves-Júnior FT, Aparício PS (2007) Estrutura fitossociológica da mata ciliar do Açude do Meio. Reserva Ecológica de Dois Irmãos, Recife-PE. Magistra. 19(1): 31-39.

Honda EA, Durigan GA (2017) Restauração de ecossistemas e a produção de água. Revista Hoehnea, 44(3): 315-327. doi: 10.1590/2236-8906$82 / 2016$

Jesus JBD, Oliveira LS, Gama DC (2018) Composição florística e caracterização do estado de conservação de nascentes no centro-leste da Bacia Hidrográfica do rio Itapicuru, semiárido da Bahia. Floresta. 4(2): 245-254. doi: 10.5380/rf.v48 i2.55540.

Leal MS, Tonello KC, Dias HCT, Mingoti R (2017) Caracterização hidroambiental de nascentes. Revista Ambiente \& Água. 12(1): 146-155. doi: 10.4136/ambi-agua.1909.

Machado LC, Selva VSF, Santos, SM (2018) Proposta metodológica interdisciplinar como ferramenta para o potencial de conservação de nascentes. Journal of Environmental Analysis and 
Progress. 3(1): 008-023. doi: 10.24221/jeap.3.1.2018.1660.008.

Magalhães LTS, Gomes JBV, Vasco AND, Netto ADOA, Ferreira RA (2012) Caracterização geopedológica das áreas de nascentes na Bacia Hidrográfica do rio Piauitinga, Sergipe, Brasil. Ambiente \& Água. 7(1): 169-181. doi: 10.4136/ambi-agua.767.

Magurran AE (1988) Ecological diversity and its measurement. Princeton University Press: New Jersey. 179p.

Marmontel CVF, Rodrigues VA (2015) Parâmetros indicativos para qualidade da água em nascentes com diferentes coberturas de terra e conservação da vegetação ciliar. Floresta e Ambiente. 22(2): 171181. doi: 10.1590/2179-8087.082014.

Oliveira DGD, Ferreira RA, Mello AAD, Oliveira RSCD, Oliveira RSCD (2012) Análise da vegetação em nascentes da Bacia Hidrográfica do rio Piauitinga, Salgado, SE. Revista Árvore, 36(1): 127141. doi: 10.1590/S0100-67622012000100014

Paula A, Silva AF, Marco-Júnior P, Santos FAM, Souza AL (2004) Sucessão ecológica da vegetação arbórea em uma Floresta Estacional Semidecidual, Viçosa, MG, Brasil.Acta Botanica Brasilica. 18(3): 407-423. doi: 10.1590/S0102-33062004000300002.

Pereira-Silva EFL, Pires JSR, Hardt E, Santos JE, Ferreira WA (2011) Avaliação da qualidade da água em microbacias hidrográficas de uma Unidade de Conservação do Nordeste do estado de São Paulo, Brasil. Revista Brasileira de Biociências. 9(3): 371381.

Pieroni JP, Branco KGR, Ferreira GC (2019) Avaliação do estado de conservação de nascentes em Microbacias Hidrográficas. Geociências. 38(1): 185-193.

Pinto LVA, Botelho AS, Davide AC, Ferreira E (2005a) Estudo da vegetação como subsídios para propostas de recuperação das nascentes da Bacia Hidrográfica do Ribeirão Santa Cruz, Lavras, MG. Scientia Forestalis. 65: 775-793.

Pinto LVA, Botelho SA, Davide AC, Ferreira E (2004) Estudo das nascentes da Bacia Hidrográfica do Ribeirão Santa Cruz, Lavras, MG. Scientia Forestalis. 65: 197-206.

Pinto LVA, Botelho SA, Oliveira-Filho AT, Davide AC (2005c) Estudo da vegetação como subsídios para propostas de recuperação das nascentes da
Bacia Hidrográfica do Ribeirão Santa Cruz, Lavras, MG. Revista Árvore. 29(5): 775-793.

Pinto LVA, Ferreira E, Botelho AS, Davide AC (2005b) Caracterização física da Bacia Hidrográfica do Ribeirão Santa Cruz, Lavras, MG e uso conflitante da terra em suas Áreas de Preservação Permanente, Cerne. 11(1): 197-206.

Pinto, LVA, Roma, TND, Balieiro, KRDC (2012) Avaliação qualitativa da água de nascentes com diferentes usos do solo em seu entorno. Cerne, 18(3): 495-505. doi:10.1590/S010477602012000300018 .

Resende HC, Mendes DR, Mendes JEDG, Bernardes WA (2009) Diagnóstico e ações de conservação e recuperação para as nascentes do Córrego-Feio, Patrocínio, MG. Bioscience Journal. 25(5): 112119.

Rodrigues ABM, Giuliatti NM, Pereira Júnior A. (2020) Aplicação de metodologias de recuperação de áreas degradadas nos biomas brasileiros Brazilian Applied Science Review.4(1): 333-369. doi: 10.34115/basrv4n1-021.

Santos TIS (2009) Estado de conservação e aspectos da vegetação de nascentes do riacho Grilo-SE. Dissertação, Universidade Federal de Sergipe-UFS, São Cristóvão, 95p.

Santos WA, Cruz JF, Almeida AQ, Mello AA, Santos RB, Loureiro DC (2017) Conflito de uso da terra em áreas de preservação permanentes da Bacia do rio Piauitinga, Sergipe, Brasil. Revista de Ciências Agrárias. 60(1): 19-24. doi: 10.4322/rca.2281.

Sergipe (2004)Atlas digital de Recursos Hídricos. SEPLAN/SRH. Aracaju-SE. CD-ROM.

Sergipe (2010) Secretaria Estadual do Meio Ambiente e dos Recursos Hídricos de Sergipe SEMARH. Bacias Hidrográfica: Plano Estadual de Recursos Hídricos - PERH. Disponível em: https://sedurbs.se.gov.br/portalrecursoshidricos/?pa gina=pro comite\#. Acesso em 23 de dezembro de 2020.

Silva PLF, Figueirêdo Neto NG, Silva BOT, Monteiro JEA, Xavier HF (2016) Degradação, uso e ocupação do solo em áreas de nascentes na Microbacia Hidrográfica do Rio Guarabira. Acta Iguаzи. 5(4): 42-53.

Siqueira MN, Morais AR, Faria KMS, Castro SS (2016) Permanent preservation areas of Mineiros, Goiás, in light of the new native vegetation protection policy - Law 12.651/2012. Revista 
Árvore. 40(4): 575-584. doi: $10.1590 / 0100-$ $\underline{67622016000400001 .}$

Sobrinho FAP, Christo AG, Guedes-Bruni RR, Silva AF (2009) Composição florística e estrutura de um fragmento de floresta estacional semidecidual aluvial em Viçosa (MG). Floresta. 39(4): 793-805. doi: 10.5380/rf.v39i4.16314.

Souza KISD, Chaffe PLB, Carvalho Pinto CRSD, Nogueira TMP (2019) Proteção ambiental de nascentes e afloramentos de água subterrânea no Brasil: histórico e lacunas técnicas atuais. Águas Subterrâneas. 33(1): 76-86. doi: 10.14295/ras.v33i1.29254.

Souza LMD, Faria RAVB, Botelho SA, Fontes MAL, Faria JMR (2012) Potencial da regeneração natural como método de restauração do entorno de nascente perturbada. Cerne. 18(4): 565-576. doi: $\underline{10.1590 / \mathrm{S} 0104-77602012000400006}$

Swaine MD, Whitmore TC (1988) On the definitions of ecological species groups in tropical forest. Vegetatio. 75(2): 81-86.

Tambosi LR, Vidal MM, Ferraz SFDB, Metzger JP (2015) Funções eco-hidrológicas das florestas nativas e o Código Florestal. Estudos avançados. 29(84): 151-162. doi: 10.1590/S010340142015000200010 .

Valle Júnior RFD, Pissarra TC, Passos ADO, Ramos TG, Abdala VL (2010) Diagnóstico das áreas de preservação permanente na Bacia Hidrográfica do Rio Tijuco, Ituiutaba-MG, utilizando tecnologia SIG. Engenharia Agrícola. 30(3): 495-503. doi: 10.1590/S0100-69162010000300013.

Vanzela ES, Hernandez FBT, Franco RAM (2010) Influência do uso e ocupação do solo nos recursos hídricos do Córrego Três Barras, Marinópolis. Revista Brasileira de Engenharia Agrícola e Ambiental. 14(1): 55-64. doi: 10.1590/S1415$\underline{43662010000100008 .}$. 